\title{
Efectos de la actividad física en el autoconcepto y la autoeficacia en preadolescentes Effects of physical activity on self-concept and self-efficacy in preadolescents
}

\author{
Tilly Louise Kyle, Antonio Hernández Mendo, Rafael Enrique Reigal Garrido, Verónica Morales Sánchez
} Universidad de Málaga (España)

Resumen. El propósito del presente trabajo fue evaluar los efectos de la práctica física regular sobre el autoconcepto y la autoeficacia en un grupo de preadolescentes. Participaron en este trabajo 63 niños y niñas de la ciudad de Málaga (España), con edades entre los 10 y los 12 años ( $M=10.54 ; D T$ =.56). Se empleó un diseño cuasi-experimental con un grupo control y otro experimental. Para evaluar el autoconcepto se implementó el Cuestionario Autoconcepto Forma 5 (AF5) y para analizar la autoeficacia se utilizó la Children's Perceived Self-efficacy Scale. Además, como medidas de control se evaluó la condición física, el tiempo dedicado al estudio y el tiempo de descanso. Los resultados encontrados indicaron efectos significativos de la práctica regular de actividad física sobre en el autoconcepto físico y el autoconcepto académico. Asimismo, se obtuvieron indicios de significación en la autoeficacia académica y la autoeficacia social. Los datos encontrados sugieren que practicar actividad física en la adolescencia puede mejorar los procesos de desarrollo de los niños y niñas influyendo en este tipo de variables.

Palabras claves. Actividad física; autoconcepto; autoeficacia; preadolescencia.

Abstract. The purpose of this study was to evaluate the effects of regular physical exercise on self-concept and self-efficacy in a group of preadolescents. The participants of this study were 63 children from the city of Málaga (Spain), aged 10 to 12 years (M = 10.54, SD = .56). A quasiexperimental design was used, including a control group and an experimental group. To assess self-concept, the Autoconcepto Forma 5 Questionnaire (AF5) was used and to analyze the self-efficacy the Self-Efficacy Scale for Children (EAN) was used. In addition, as control measures, physical fitness, studying and sleeping hours were assessed. The results indicated significant effects of regular physical activity on physical self-concept and academic self-concept. Also, evidence of significant effects was obtained in academic self-efficacy and social self-efficacy. This data suggests that practicing physical activity in preadolescence may improve development processes, having an effect on children in the variables investigated.

Key words. Physical activity; self-concept; self-efficacy; preadolescence.

\section{Introducción}

Un amplio conjunto de investigaciones han demostrado los beneficios que tiene la práctica de actividad física regular en diversas etapas de la vida, tanto a nivel físico como psicológico y social (Annesi, 2010; Chen \& Lee, 2013; Owen, Salmon, Koohsari, Turrell \& Giles-Corti, 2014; Zschucke, Gaudlitz \& Ströhle, 2013). Específicamente, existen estudios que han puesto de relieve la importancia de promocionar estilos de vida activos en la infancia y la adolescencia dada su repercusión sobre la salud y el bienestar (Mota, et al., 2012; Pontifex, et al., 2011; Vedul-Kjelsäs, Sigmundsson, Stensdotter \& Haga, 2011). Esta circunstancia es especialmente relevante, dado que se trata de etapas críticas en el desarrollo de una persona y determinantes para su evolución futura, lo que puede influir de manera sustancial en la vida adulta (Blakemore \& Mills, 2014; Mills, Lalonde, Clasen, Giedd \& Blakemore, 2014; Raudino, Fergusson \& Horwood, 2014).

Son numerosas las variables que pueden repercutir en el bienestar infantil y adolescente. Desde una perspectiva psicosocial, los juicios efectuados sobre la capacidad, el valor personal y otros similares son determinantes para un buen ajuste psicológico así como para una adecuada relación con el entorno en estas edades. Por ello, el análisis de variables como la autopercepción de salud, el autoconcepto, la autoeficacia o la autoestima es especialmente interesante para poder interpretar correctamente lo que ocurre en estos periodos (Caprara, Vecchione, Alessandri, Gerbino \& Barbaranelli, 2011; Jerdén, Burell, Stenlund, Weinehall \& Bergström, 2011; Spilt, Lier, Leflot, Onghena \& Colpin, 2014). Concretamente, el autoconcepto y la autoeficacia son dos de los constructos que más atención han recibido desde diferentes perspectivas en los últimos años (Parschau, et al., 2013; Viholainen, Aro, Purtsi, Tolvanen \& Cantell, 2014; Wilson, Siegle, McCoach, Little \& Reis, 2014).

El autoconcepto se refiere a la percepción que tienen las personas de sí mismas, siendo uno de los factores psicosociales implicados en el desarrollo de los niños y los adolescentes (Coelho, Sousa \& Figueira, 2014; Delgado, Inglés \& García-Fernández, 2013). El desarrollo de este constructo está relacionado con las atribuciones que las personas efectúan sobre sí mismos, así como el resultado de las experiencias con los demás (Shavelson, Hubner \& Stanton, 1976). Un buen autoconcepto se

Fecha recepción: 24-12-14- Fecha envío revisores: 24-12-14- Fecha de aceptación: 05-07-15 Rafael Enrique Reigal Garrido rafareigal@gmail.com asocia a un adecuado funcionamiento personal y social, estando implicado en la relación con el entorno, en la adopción de conductas saludables y determinando el éxito que puede alcanzar en ámbitos tan importantes como el académico, social o deportivo (Guillén \& Ramírez, 2011; Slutzky \& Simpkins, 2009). Actualmente, el modelo de autoconcepto más extendido contempla una naturaleza multidimensional en la que se puede diferenciar sus dimensiones física, emocional, familiar, académica y social (Esnaola, Rodríguez \& Goñi, 2011; Shavelson et al., 1976).

La autoeficacia, por su parte, se puede definir como los juicios que posee cada individuo sobre sus propias capacidades, considerándolos fundamentales en la organización y ejecución de sus actuaciones (Bandura, 1986; Galicia-Moyeda, Sánchez-Velasco \& Robles-Ojeda, 2013). Estas evaluaciones son determinantes en la elección de una tarea que se va a llevar a cabo, el nivel de esfuerzo empleado, la persistencia o la forma con la que se afrontan las dificultades (Bandura, 1997). La construcción de la percepción de eficacia es un proceso complejo en el que intervienen aspectos como el éxito previo, la experiencia vicaria, la persuasión verbal o los estados fisiológicos manifestados (Bandura, 1986; Weinberg \& Stockham, 2000). Tal y como ocurre con el autoconcepto, se ha observado la importancia de este constructo en las edades infantil y adolescente en contextos como la educación, la salud o el deporte (Caserta, Wyman, Wang, Moynihan, \& O’Connor, 2011; Kane, et al., 2013; Zimmerman, 2000).

Existen estudios que han asociado la práctica de actividad física a un mejor desarrollo del autoconcepto y la autoeficacia en niños y adolescentes (e.g., Atienza, Balaguer, García-Merita, \& Moreno, 1999; Goñi, \& Zulaica, 2000; Goñi, \& Infante, 2010; Molero, Ortega, Valiente, \& Zagalaz, 2010). Como ejemplo, los trabajos de Esnaola y Revuelta (2009) o Contreras, Fernández, García, Palou, y Ponseti (2010) realizados con adolescentes pusieron de manifiesto mejores percepciones del autoconcepto físico y general en aquellos que practicaban actividad física regularmente. De igual forma, Candel, Olmedilla y Blas (2008) en un estudio con adolescentes observaron mejores puntuaciones en autoconcepto académico, social, familiar y físico en aquellos que practicaban actividad física frecuentemente. A su vez, Annesi (2006) efectuó un trabajo en el que señaló efectos positivos de un programa de actividad física sobre el autoconcepto físico y la autoeficacia en preadolescentes. Asimismo, Bean, Miller, Mazzeo y Fries (2012) indicaron que la participación en actividades físicas tenía un efecto positivo sobre la autoeficacia física en un grupo de chicas adolescentes.

Cuando se analiza el impacto de la actividad física sobre variables de salud física y psicosocial se pueden atender a diferentes parámetros 
para explicar los efectos producidos. En este sentido, existen autores que ponen de relieve la importancia de analizar la condición física desarrollada para explicar mejor la relación entre la práctica física regular y sus beneficios (Carraro, Scarpa \& Ventura, 2010; García-Artero, et al., 2007; Martins, et al., 2010; Ramírez-Lechuga, Femia, Sánchez-Muñoz \& Zabala, 2011). En esta línea, Crocker, Sabiston, Kowalski, McDonough y Kowalski (2006) realizaron un estudio con adolescentes observando que el índice de masa corporal estaba relacionado con el autoconcepto general y físico. En otra investigación efectuada por Velez, Golem y Arent (2010) sobre un grupo de adolescentes indicó que un programa de actividad física, basado en entrenamiento de resistencia generaba mejoras en los niveles de autoconcepto físico y general. Asimismo, Dunton, Schneider y Cooper (2007) en un trabajo con adolescentes pusieron de relieve que mejoras en la capacidad aeróbica estaban relacionadas con una percepción más positiva de la autoeficacia general. Pesce, Faigenbaum, Crova, Marchetti y Bellucci (2013) analizaron los efectos de dos programas de actividad física en preadolescentes y observaron incrementos en autoeficacia social en el grupo que tuvo mayores incrementos de la capacidad aeróbica.

En base a los antecedentes descritos, la presente investigación analiza los efectos de la práctica física en el autoconcepto y la autoeficacia en un grupo de preadolescentes. Asimismo, evalúa los cambios producidos en diversas pruebas de condición física para mostrar si existían cambios diferenciales entre aquellos que practicaban o no actividad física regular.

\section{Método}

\section{Muestra}

Participó en la investigación una muestra incidental de 63 preadolescentes de la ciudad de Málaga (España), con edades entre los 10 y 12 años $(M \pm D T$ : edad $=10.54 \pm .56$ años; altura $=148.32 \pm 7.08$ $\mathrm{cm}$; peso $=45.59 \pm 9.38 \mathrm{~kg}$; IMC $=20.70 \pm 3.34 \mathrm{~kg} \cdot \mathrm{m}^{-2}$ ). Se tuvieron en cuenta como criterios de exclusión tener problemas de salud que pudieran afectar a la investigación y no presentar el consentimiento informado firmado por los padres. Tras recoger los consentimientos informados, los alumnos cumplimentaron el cuestionario «ad hoc» sobre la actividad física realizada, tiempo de descanso y tiempo de estudio a los participantes que si podían realizar el estudio. Esto sirvió para luego organizar a los participantes en función de las horas de actividad física realizada. Se organizaron en dos grupos, un grupo control (no practicaban actividad física regular en horario extraescolar o la realizaban de manera ocasional como máximo una vez a la semana; $n=32,13$ chicos y 19 chicas) y un grupo experimental (sí participaban en programas de actividad física estructurada o practicaban habitualmente dos o más días a la semana; $n=31,17$ chicos y 14 chicas).

\section{Medidas}

a) Autoconcepto. Para evaluar este constructo se utilizó el Cuestionario Autoconcepto Forma 5 (AF5 - García \& Musitu, 2001). Este cuestionario, formado por 30 ítems, evalúa el autoconcepto multidimensional, estando formado por 5 factores: académico (e.g., Hago bien los trabajos escolares), social (e.g., Hago fácilmente amigos), emocional (e.g., Tengo miedo de algunas cosas), familiar (e.g., Soy muy criticado en casa) y físico (e.g., Me cuido físicamente). Se contesta mediante una escala con puntuaciones entre uno y 99, en el que 1 significa estar en total desacuerdo y 99 un acuerdo máximo con lo dictado en cada cuestión. Posteriormente esta escala se traslada a una más reducida con puntuaciones entre uno y 10 para su análisis e interpretación. La aplicación de este cuestionario se ubica en un espectro de población muy amplio, desde la infancia hasta la edad adulta. Los análisis de fiabilidad (Alfa de Cronbach), en este trabajo y para cada subescala, ofrecen puntuaciones adecuadas (entre .71 y .85).

b) Autoeficacia. Para analizar la percepción de eficacia se empleó la Children's Perceived Self-efficacy Scale (Bandura, 1990; Pastorelli, et al., 2001) en su adaptación a población española (Escala deAutoeficacia para Niños, EAN; Carrasco, \& Del Barrio, 2002). Constituido por 35 ítems, se estructura en 3 factores: autoeficacia académica (e.g., Quétal se te dan las matemáticas?), autoeficacia social (e.g., ¿Qué tal se te da participar en debates en clase?) y autoeficacia autorregulatoria (e.g., ¿Quétal se te dan las actividades deportivas?). Se contesta mediante una escala likert de uno a cinco ( 1 = fatal, 2 = no muy bien, $3=$ = bien, $4=$ muy bien y 5 = fenomenal). Los análisis de fiabilidad (Alfa de Cronbach), en este trabajo y para cada subescala, ofrecen puntuaciones adecuadas (entre .74 y .91).

c) Práctica física, tiempo de descanso y tiempo de estudio. Se utilizó un cuestionario elaborado «ad hoc» para analizar la práctica física realizada, el tiempo de descanso y el tiempo de estudio. En él, se preguntaba si se realizaba práctica física en el tiempo libre (Sí / No), la frecuencia de práctica (1 día por semana/2 a 4 días por semana/ 5 o más días por semana) y el tipo de práctica realizada. Además, se preguntó por el tiempo dedicado regularmente al estudio y al descanso (durante periodo lectivo pero no coincidente con el final de trimestre escolar). El valor utilizado para el estudio fue el valor medio semanal. El objetivo de evaluar el tiempo de descanso y estudio era analizar posibles variables contaminantes.

d) Condición física. Se evaluó de forma indirecta el consumo máximo de oxígeno(VO2máx) a través del test de 1000 metros, utilizando la fórmula VO2máx $(\mathrm{ml} / \mathrm{kg} / \mathrm{min})=74.8665$ - $6.5125 * \mathrm{t}$ (tiempo en minutos) + E (error estándar de predicción) (Melchor, Montaño, Díaz \& Cervantes, 2013). Además se efectuó el test de salto horizontal para evaluar la fuerza explosiva en los miembros inferiores (Eurofit, 1993). Asimismo se evaluó la velocidad a través de una prueba «ad hoc» en la que tenían que recorrer tres veces una distancia de 20 metros (prueba de ida y vuelta).

\section{Procedimiento}

Para realizar este trabajo se contactó con el centro escolar y se solicitó permiso a la dirección del centro para efectuar la investigación. Posteriormente, se obtuvo consentimiento informado de los padres o tutores legales para que los alumnos/as pudieran participar. Además, durante todo el proceso de investigación se respetaron los principios establecidos en la declaración de Helsinki (2000).

En el presente estudio se realizó una evaluación en dos momentos temporales distintos. Entre ambas mediciones, los participantes asignados al grupo experimental estuvieron involucrados en programas de actividad estructurada, generalmente entre dos y cuatro días de práctica física semanal. Por otro lado, los integrantes del grupo control no realizaron ningún tipo de actividad física o manifestaban hacerla ocasionalmente como máximo una vez a la semana. El intervalo total del estudio fue de siete meses, desde la evaluación pre-test realizada en noviembre de 2013 hasta la evaluación post-test en mayo 2014.

Cada medición se efectuó a lo largo de una semana. Los cuestionarios fueron autoadministradas aunque estuvo presente un investigador para resolver posibles dudas. Las evaluaciones se realizaron en condiciones de silencio absoluto y en un lugar adaptado para ello. Por otro lado, el profesor de educación física realizó la evaluación de la condición física de los participantes.

\begin{tabular}{|c|c|c|c|c|c|c|c|c|c|c|}
\hline & \multicolumn{5}{|c|}{ Grupo control } & \multicolumn{5}{|c|}{ Grupo experimental } \\
\hline & $M$ & $D T$ & $A$ & $K$ & $Z$ & $M$ & $D T$ & $A$ & $K$ & $Z$ \\
\hline \multicolumn{11}{|c|}{ Velocidad (seg.) } \\
\hline Pre & 17.56 & 1.49 & .24 & 1.40 & .69 & 18.21 & 1.58 & -13 & -09 & .72 \\
\hline Post & 16.92 & 1.29 & -.33 & -.58 & .64 & 17.11 & 1.38 & -13 & .39 & .46 \\
\hline \multicolumn{11}{|c|}{ Sal to (cm.) } \\
\hline Pre & 131.28 & 19.16 & -1.03 & .47 & .91 & 129.55 & 18.94 & -34 & .63 & .71 \\
\hline Post & 134.72 & 18.57 & -.81 & .06 & .81 & 135.61 & 17.24 & .52 & .91 & .90 \\
\hline \multicolumn{11}{|c|}{ VO2máx (ml/kg/min.) } \\
\hline Pre & 41.68 & 3.76 & -1.03 & .64 & 1.01 & 40.85 & 3.56 & -87 & 1.16 & .64 \\
\hline Post & 42.50 & 3.59 & -1.16 & .63 & 1.12 & 42.19 & 3.11 & -1.01 & 1.20 & .76 \\
\hline \multicolumn{11}{|c|}{ Descanso } \\
\hline Pre & 556.29 & 49.75 & -.74 & .49 & .87 & 564.84 & 54.38 & -.62 & .01 & .89 \\
\hline Post & 552.48 & 40.09 & -.82 & .57 & .85 & 562.61 & 49.49 & -.54 & -.04 & .73 \\
\hline \multicolumn{11}{|c|}{ Estudio } \\
\hline Pre & 134.52 & 62.87 & .30 & -.68 & 1.14 & 109.35 & 56.15 & .43 & -.47 & .75 \\
\hline Post & 132.03 & 71.69 & .86 & .13 & .99 & 111.58 & 55.17 & .67 & .05 & .83 \\
\hline
\end{tabular}




\section{Análisis de datos}

Se realizó un análisis descriptivo de los datos. La normalidad de las variables fue analizada utilizando el test de Kolmogorov-Smirnov. El test de Levene fue realizado para analizar el supuesto de homogeneidad de varianzas entre grupos. Se realizó un análisis de la varianza (ANOVA) bifactorial para cada medida de condición física y autoconcepto y autoeficacia, siendo un primer factor el grupo con dos niveles (control y experimental) y un segundo factor la variable pre-post con dos niveles (valores previos y tras la intervención). Se estudiaron los efectos principales y la interacción entre variables, utilizándose el estadístico de Bonferroni para evaluar la significación. Todos los análisis se realizaron utilizando el programa estadístico (SPSS v.20).

\section{Resultados}

En la tabla 1 se muestran los estadísticos descriptivos y de normalidad para las medidas de condición física, tiempo de descanso y tiempo de estudio. Los resultados indicaron que los datos presentaban distribución normal.

En la tabla 2 se muestran los resultados de los ANOVAs factoriales mixtos realizados para las medidas de la tabla 1 . Como se puede observar, hubo significación en los efectos principales de las variables prepost para todas las medidas salvo para descanso y estudio, aunque no en los efectos de la variable grupo. Síse observaron efectos de interacción significativos para los velocidad, salto y resistencia. Además, la prueba de Levene indicó que existía homogeneidad de varianza en cada medida y grupo $(p>.05)$. Asimismo, la prueba de Levene indicó que existía homogeneidad de varianza en cada medida y grupo $(p>.05)$.

Tabla 2 .

Resultados de los ANOV As factoriales mixtos para las medidas de condición física, tiempo de descanso y estudio. Se muestran los val ores de la $F$, el valor $p$, el tamaño del efecto $\left(\eta^{2}\right)$ y la potencia del contras te $(1-\beta)$

\begin{tabular}{|c|c|c|c|c|}
\hline & & Pre-post & Grupo & Interacción \\
\hline \multirow{3}{*}{ Velocidad } & F & $110.42 * * * *$ & 1.40 & $7.52^{* * *}$ \\
\hline & $\eta^{2}$ & .64 & .02 & .11 \\
\hline & $1-\beta$ & .99 & .22 & .77 \\
\hline \multirow{3}{*}{ Salto } & $F$ & $81.83^{* * *}$ & .01 & $6.23^{*}$ \\
\hline & $\eta^{2}$ & .57 & .01 & .09 \\
\hline & $1-\beta$ & .99 & .05 & .69 \\
\hline \multirow{3}{*}{ VO2máx } & $F$ & $71.79 * * *$ & .42 & $4.20^{*}$ \\
\hline & $\eta^{2}$ & .54 & .01 & .06 \\
\hline & $1-\beta$ & .99 & .10 & .52 \\
\hline \multirow{3}{*}{ Descanso } & $F$ & .24 & .76 & .02 \\
\hline & 72 & .01 & .01 & .01 \\
\hline & $1-\beta$ & .08 & .14 & .05 \\
\hline \multirow{3}{*}{ Estudio } & $F$ & .01 & 2.51 & .14 \\
\hline & $\eta 2$ & .01 & .04 & .01 \\
\hline & $1-\beta$ & .05 & .34 & .07 \\
\hline
\end{tabular}

Tabla 3

\begin{tabular}{lcccc}
\multicolumn{5}{c}{ Comparaciones entregrupos e intragrupos para cada prueba de condición física } \\
\cline { 2 - 5 } & Control & Grupo & Experimental & Factor \\
\cline { 2 - 5 } & Pre vs. Post & Pre vs. Post & C vs. E & Postest \\
\hline Velocidad & $.64^{* * * *}$ & $1.10^{* * * *}$ & -.65 & -.19 \\
Sal to & $-3.44^{* * *}$ & $-6.06^{* * * *}$ & 1.73 & -.89 \\
VO2max & $-.82^{* * * *}$ & $-1.35^{* * * *}$ & .83 & .31 \\
\hline \multicolumn{5}{c}{$* * 01$} \\
\multicolumn{5}{c}{ VO2max= Consumo de oxígeno máximo }
\end{tabular}

Tabla 4.

Medias, desviaciones típicas y prueba de normalidad (Kolmogorov-Smirnov) de los valores de autoconcepto y autoeficacia

\begin{tabular}{|c|c|c|c|c|c|c|c|c|c|c|}
\hline & & & po cont & & & & Grupo & xperim & & \\
\hline & $M$ & $D T$ & $A$ & $K$ & $Z$ & $M$ & $D T$ & $A$ & $K$ & $Z$ \\
\hline $\mathrm{ACA}$ & & & & & & & & & & \\
\hline Pre & 8.52 & 1.21 & -.51 & \begin{tabular}{c|c|}
-1.08 \\
\end{tabular} & $\begin{array}{l}.78 \\
\end{array}$ & 7.77 & 1.74 & -91 & .21 & .95 \\
\hline Post & 8.04 & 1.47 & -1.13 & .48 & .95 & 8.40 & 1.35 & -95 & .20 & .74 \\
\hline AC So & & & & & & & & & & \\
\hline Pre & 8.00 & 1.25 & -.18 & -.69 & .45 & 7.88 & 1.53 & -1.28 & 1.52 & 1.06 \\
\hline Post & 7.62 & 1.51 & -.05 & -.94 & .48 & 8.04 & 1.32 & -1.05 & 1.59 & .83 \\
\hline $\mathrm{AC} \mathrm{En}$ & & & & & & & & & & \\
\hline Pre & 4.92 & 2.41 & .03 & -1.20 & .73 & 4.81 & 2.43 & .33 & -.74 & .58 \\
\hline Post & 5.04 & 1.83 & .15 & -.56 & .53 & 4.56 & 2.44 & .42 & -85 & .76 \\
\hline $\mathrm{AC} \mathrm{Fa}$ & & & & & & & & & & \\
\hline Pre & 9.16 & 88 & -1.34 & 1.60 & 1.14 & 9.19 & .77 & -1.18 & .84 & 1.05 \\
\hline Post & 9.14 & 1.00 & -1.54 & 1.35 & 1.33 & 9.38 & .74 & -1.55 & 1.20 & 1.34 \\
\hline AC Fi & & & & & & & & & & \\
\hline Pre & 7.78 & 1.70 & -1.17 & .92 & .98 & 7.83 & 1.59 & -40 & -1.02 & .58 \\
\hline Post & 7.25 & 1.69 & -1.23 & 1.56 & .75 & 8.24 & 1.36 & -90 & .77 & .64 \\
\hline $\mathrm{AE} \mathrm{Ac}$ & & & & & & & & & & \\
\hline Pre & 4.09 & .49 & -.45 & .03 & .62 & 3.92 & 2.66 & -1.11 & 95 & .73 \\
\hline Post & 3.90 & .56 & -.73 & .77 & .63 & 4.04 & .57 & -89 & .87 & .70 \\
\hline AE So & & & & & & & & & & \\
\hline Pre & 4.14 & .51 & -.40 & -.98 & .76 & 4.19 & .59 & -66 & -47 & .89 \\
\hline Post & 4.09 & .61 & -.94 & .50 & .86 & 4.41 & .44 & -1.38 & 1.59 & 1.12 \\
\hline $\mathrm{AE} \operatorname{Re}$ & & & & & & & & & & \\
\hline Pre & 4.28 & .66 & -.90 & -.12 & 1.04 & 4.35 & .67 & -1.20 & 1.01 & .93 \\
\hline Post & 4.33 & .54 & -1.17 & 1.68 & 1.05 & 4.32 & .70 & -1.08 & .10 & 1.31 \\
\hline
\end{tabular}

Tabla 5.

Resultados de los ANOVAs factoriales mixtos para las medidas de autoc oncepto y autoe ficacia. Se muestran los valores de la $F$, el valor $p$, el tan

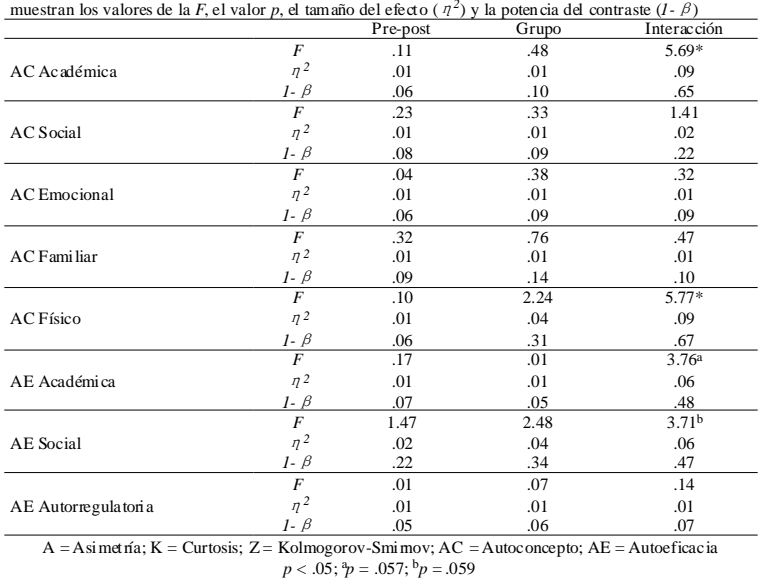

En la tabla 3 se muestran las comparaciones entre grupos e intragrupos de las medidas de condición física. Como se puede observar, no existieron diferencias pretest ni postest. Tanto el grupo control como el experimental mejoraron en las pruebas de condición física, siendo superior el cambio en el grupo experimental.

En la tabla 4 se muestran los estadísticos descriptivos y pruebas de normalidad de las diferentes escalas de autoconcepto y autoeficacia. Los resultados indicaron que los datos presentaban distribución normal.

La tabla 5 muestra los ANOVAs factoriales mixtos efectuados. Como se puede observar, no hubo significación en los efectos principales de las variables pre-post y grupo. Sí se observaron efectos de interacción significativos para los factores autoconcepto académico y autoconcepto físico, así como indicios de significación en autoeficacia académica y autoeficacia social.Además, la prueba de Leveneindicó que existía homogeneidad de varianza en cada medida y grupo ( $p>.05)$.
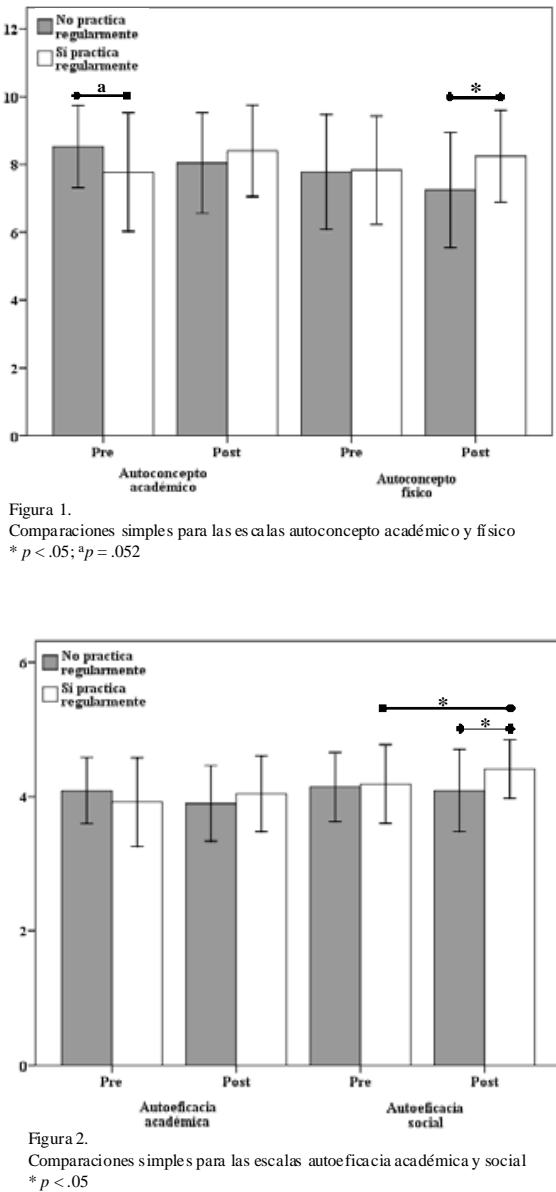
En las figuras 1 y 2 se pueden observar las comparaciones simples entre los grupos e intragrupos de los factores cuya interacción ha sido significativa (o próxima a la significación). Tal y como se muestra, no hubo en ningún caso diferencias entre las medidas pretest aunque sí indicios de significación entre grupos en autoconcepto académico. En las postest sí hubo diferencias significativas en autoconcepto físico y autoeficacia social. Por otro lado, en el grupo control no hubo diferencias entre las medidas pre y post, y sí en el grupo experimental tanto en autoeficacia social.

\section{Discusión}

Este trabajo pretendía analizar los efectos de la actividad física regular sobre el autoconcepto y la autoeficacia en una muestra preadolescente. Los resultados han puesto de manifiesto efectos significativos sobre el autoconcepto académico y físico, así como indicios de significación en autoeficacia académica y social, lo que satisface el objetivo de la investigación. Este estudio se sitúa en la línea de otros trabajos que habían señalado los beneficios de la práctica física en la infancia y la adolescencia en diversos parámetros psicosociales (Dunton, Schneider, Graham \& Cooper, 2006; Vedul-Kjelsäs, et al., 2011).

Específicamente, esta investigación se encuentra en consonancia con otros trabajos que habían observado efectos positivos de la práctica regular de ejercicio físico sobre diversas dimensiones del autoconcepto y autoeficacia en estas edades (Annesi, 2006; Bean, etal., 2012; Contreras, et al., 2009; Esnaola \& Revuelta, 2009). No obstante, es necesario destacar el comportamiento intragrupo de cada medida, dado que en el grupo experimental únicamente hubo diferencias significativas entre las medidas iniciales y finales en autoeficacia social, existiendo en las demás variables incrementos aunque no significativos. Así, los efectos de interacción se han debido, en algunos casos, a un leve descenso en los valores del grupo control. En cualquier caso, esto refuerza el valor de la práctica física al sugerir que podría funcionar como un factor de protección en una etapa de cierta vulnerabilidad (Mills, et al., 2014; Raudino, et al., 2014).

También se han evaluado diferentes variables de condición física, indicando incrementos más acusados en el grupo que practicaba actividad física regularmente. La evolución paralela de estas medidas y las de tipo psicosocial es coherente con otros trabajos que habían señalado la relación existente entre ellas (Crocker, et al., 2006; Dunton, et al., 2007; Pesce, et al., 2013; Velez, et al., 2010). Aunque pueden existir diferentes factores que influyan en la mejora del autoconcepto o la autoeficacia en practicantes de ejercicio físico, el incremento de la condición física permite considerar que un aumento en la percepción de competencia física o la percepción de la imagen corporal sería uno ellos dada su importancia en estas edades. Además, existen diversos autores que sugieren que la evaluación de la condición física explica mejor los beneficios asociados a la práctica física (Carraro, et al., 2010; Martins, et al., 2010; RamírezLechuga, et al., 2011). Por lo tanto, el simple hecho de practicar actividad física no debería ser la única medida para determinar los beneficios sobre la salud, dado que el impacto que genere puede depender de los efectos que cause sobre el propio organismo (Ortega, Ruiz, Castillo \& Sjöström, 2007).

Asimismo, se efectuó un control del tiempo de estudio y descanso, para evaluar sin podría existir algún elemento que pudiera moldear los resultados obtenidos. Tanto en el tiempo de estudio como de descanso no se observaron cambios significativos, lo que indica que no se han modificado sustancialmente estos hábitos a lo largo del estudio. Sí se detectaron ligeras diferencias en las medidas iniciales en autoconcepto académico, que podrían deberse a un mayor tiempo de estudio por parte del grupo control. Sin embargo, la evolución de esta medida no es congruente con el tiempo dedicado por cada grupo al tiempo de estudio, lo que sugiere que los cambios producidos podrían deberse a otras circunstancias. De hecho, existen evidencias que han puesto de relieve beneficios del ejercicio físico sobre el funcionamiento cognitivo y el rendimiento académico en niños, lo cual podría estar asociado a una mejor percepción de las capacidades para hacer frente a la tarea académi-
ca(Chaddock, Pontifex, Hillman \& Kramer, 2011; Tomporowski, Davis, Miller \& Naglieri, 2008).

Este trabajo presenta una serie de limitaciones que se deben valorar para interpretar los resultados adecuadamente. En primer lugar el tiempo durante el cual se ha desarrollado no ha sido muy extenso, lo que podría haber sesgado la significación de los datos encontrados. En futuros trabajos se debería ampliar el periodo de investigación, efectuado mediciones intermedias para analizar también posibles fluctuaciones significativas a lo largo del mismo. Por otro lado, el grupo experimental estuvo constituido por preadolescentes que practicaban actividad física con una frecuencia variable. Se sugiere en trabajos posteriores establecer diversos grupos experimentales para comparar si se producen diferentes efectos en función del tipo de práctica llevada a cabo. Por último, el tamaño de la muestra no es representativo de la población objeto de estudio, por lo que se sugiere incrementar el número de participantes en futuros trabajos.

En cualquier caso, el presente estudio señala que ha existido un efecto positivo de la práctica física regular en la muestra analizada sobre el autoconcepto y la autoeficacia. Por ellos, este trabajo incrementa las evidencias sobre la relación entre actividad física, condición física y este tipo de autoevaluaciones. De igual modo, refuerza la necesidad de promocionar estilos de vida activos en la infancia y adolescencia para mejorar el bienestar y el desarrollo de las personas en estas etapas de la vida.

\section{Referencias}

Annesi,J.J.(2006). Relations of physical self-conceptand self-efficacy with frequency of voluntary physical activity in preadolescents: implications for after-school care programming. Journal of Psychosomatic Research, 61(4), 515-520. doi: 10.1016/j.jpsychores.2006.04.009

Annesi, J.J.(2010). Relationship of physical activity and weight loss in women with Class II and Class III obesity: Mediation of exercise-induced changes in tension and depression. International Journal of Clinical and Health Psychology, 10(3), 435-444.

Atienza, F.; Balaguer, I.; García-Merita, M.L., \& Moreno, Y.(1999). Análisis de las diferencias individuales entre chicos y chicas adolescentes en la práctica regular de actividad física en función de la autopercepción física. EnF. Guillén (ed.): $L a$ psicología del deporte en España al final del milenio (pp. 613-621). Madrid: Taravilla.

Bandura, A. (1986). Social foundations of thought and action: A Social Cognitive Theory. Englewood Cliffs, NJ: Prentice Hall.

Bandura, A. (1990). Multidimensional scales of perceived self-efficacy. Stanford University, Stanford, C.A.

Bandura, A. (1997). Self-efficacy: The exercise of Control. New York: Freeman.

Bean, M.K.; Miller, S.; Mazzeo, S.E., \& Fries, E.A. (2012). Social cognitive factors associated with physical activity in elementary school girls. American Journal of Health Behavior, 36(2), 265-274. doi: 10.5993/AJHB.36.2.11

Blakemore, S.J., \& Mills, K.L. (2014). Is adolescence a sensitive period for sociocultural processing?. Annual Review of Psychology, 65, 187-207. doi: 10.1146/annurev-psych-010213-115202

Candel, N.; Olmedilla, A., \& Blas, A. (2008). Relaciones entre la práctica de actividad física y el autoconcepto, la ansiedad y la depresión en chicas adolescentes. Cuadernos de Psicología del Deporte, 8(1), 61-77.

Caprara, G.V.; Vecchione, M.; Alessandri, G.; Gerbino, M., \& Barbaranelli, C. (2011). The contribution of personality traits and self-efficacy beliefs to academic achievement:Alongitudinal study. British Journal of Educational Psychology, 81(1), 78-96. doi: 10.1348/2044-8279.002004

Carraro, A.; Scarpa, S., \& Ventura, L. (2010). Relationships between physical selfconcept and physical fitness in italian adolescents. Perceptual and Motor Skills, 110(2), 522-530. doi: 10.2466/pms.110.2.522-530

Carrasco, M.A., \& Del Barrio, M.V.(2002). Evaluación de la autoeficacia en niños y adolescentes. Psicothema, 14(2), 323-332.

Caserta, M.T.; Wyman, P.A.; Wang, H.; Moynihan, J., \& O’Connor, T. G. (2011). Associations among depression, perceived self-efficacy, and immune function and healthinpreadolescent children. Development and Psychopathology, 23(04), 1139-1147. doi: 10.1017/S0954579411000526

Chaddock, L.; Pontifex, M.B.; Hillman, C.H., \& Kramer, A.F. (2011). Areview of the relation of aerobic fitness and physical activity to brain structure and function in children. Journal of the International Neuropsychological Society, 17(6), 111. doi: $10.1017 / \mathrm{S} 1355617711000567$

Chen, J.J., \& Lee, Y. (2013). Physical Activity for Health: Evidence, Theory, and Practice. Journal of Preventive Medicine and Public Health, 46(Suppl 1), S1- 
S2. doi: 10.3961/jpmph.2013.46.S.S1

Coelho, V.; Sousa, V., \& Figueira,A.P. (2014). O impacto de um programa escolar de aprendizagem socioemocional sobre o autoconceito de alunos de $3^{\circ}$ ciclo. Revista de Psicodidáctica, 19(2), 347-365. doi: 10.1387/RevPsicodidact.10714

Contreras, O.R.; Fernández, J.G.; García, L.M.; Palou, P., \& Ponseti, J. (2010). E autoconcepto físico y su relación con la práctica deportiva en estudiantes adolescentes. Revista de Psicología del Deporte, 19(1), 23-39.

Crocker, P.R.E.; Sabiston, C.M.; Kowalski, K.C.; McDonough, M.H., \& Kowalski, N. (2006). Longitudinal assessment of the relationship between physical selfconcept and health-related behavior and emotion in adolescent girls. Journal of Applied Sport Psychology, 18(3), 185-200. doi: 10.1080/10413200600830257

Delgado, B.; Inglés, C.J., \& García-Fernández, J.M. (2013). Social anxiety and selfconcept in adolescence. Revista de Psicodidáctica, 18(1),179-194. doi: 10.1387/ RevPsicodidact.6411

Dunton, G.F.; Schneider, M.; Graham, D.J., \& Cooper, D.M. (2006). Physical Activity, Fitness, and Physical self-Concept in Adolescent Females. Pediatric Exercise Science, 18(2), 240-251.

Dunton, GF.; Schneider, M., \& Cooper, D.M.(2007).An investigation of psychosocia factors related tochanges in physical activity and fitness among femaleadolescents. Psychology and Health, 22(8), 929-944. doi: 10.1080/14768320601124915

Esnaola, I., \& Revuelta, L. (2009). Relaciones entre la actividad física, autoconcepto físico, expectativas, valor percibido y dificultad percibida.Acción Psicológica, 6(2), 31-43. doi: 10.5944/ap.6.2.219

Esnaola, I.; Rodríguez, A., \& Goñi, E. (2011). Propiedades psicométricas de cuestionario de Autoconcepto AF5. Anales de Psicología, 27(1), 109-117.

Eurofit (1993). Eurofit Tests of Physical Fitness ( $2^{\mathrm{a}}$ ed.). Strasbourg: Committee of Experts on Sports Research.

García, F., \& Musitu, G. (2001). Autoconcepto Forma 5. AF5. Manual. Madrid: TEA.

Galicia-Moyeda, I.X.; Sánchez-Velasco,A., \& Robles-Ojeda,F.J.(2013).Autoeficacia en escolares adolescentes: su relación con la depresión, el rendimiento académico y las relaciones familiares. Anales de Psicología, 29(2), 491-500. doi: 10.6018/analesps.29.2.124691

García-Artero, E.; Ortega, F.B.; Ruiz, J.R.; Mesa, J.L.; Delgado, M.; GonzálezGross, M. ..., \& Castillo, M.J. (2007). El perfil lipídico-metabólico en los adolescentes está más influido por la condición física que por la actividad física (estudio AVENA). Revista Española de Cardiología, 60(6), 581-588.

García-Hermoso, A.; Escalante, Y.; Domínguez, A.M., \& Saavedra, J.M. (2013) Efectos de un programa de ejercicio físico durante tres años en niños obesos: un estudio de intervención. Retos: Nuevas Tendencias en Educación Física, Deporte y Recreación, 23, 10-13.

Goñi, E., \& Infante, G. (2010). Actividad físico-deportiva, autoconcepto físico y satisfacción con la vida. European Journal of Education and Psychology,3(2), 199-208. doi: 10.1989/ejep.v3i2.60

Goñi, A., \& Zulaica, L.M. (2000). La participación en el deporte escolar y el autoconcepto en escolares de 10 a 11 años de la provincia de Guipúzcoa. Apunts, 59, 6-10.

Guillén, F., y Ramírez, M. (2011). Relación entre el autoconcepto y la condición física en alumnos del Tercer Ciclo de Primaria. Revista de Psicología del Deporte, 20(1), 45-59.

Jerdén, L.; Burell, G; Stenlund, H.; Weinehall, L., \& Bergström, E. (2011). Gender differences and predictors of self-rated health development among Swedish adolescents. Journal of Adolescent Health, 48(2), 143-150. doi: 10.1016 j.jadohealth.2010.06.005

Kane, I.; Robertson, R.J.; Fertman, C.I.; Nagle, E.F.; McConnaha,W.R., \& Rabin B.S. (2013). Self-efficacy and enjoyment of middle school children performing the progressive aerobic cardiovascular endurance run (pacer). Perceptual \& Motor Skills, 117(2), 470-483. doi: 10.2466/29.25.PMS.117x23z3

Martins, C.L.; Silva, F.; Gaya, A.R.; Aires, L.; Ribeiro, J.C., \& Mota, J. (2010) Cardiorespiratory fitness, fatness, and cardiovascular disease risk factors in children and adolescents from Porto. European Journal of Sport Science, 10(2), 121-127. doi: 10.1080/17461390903307842

Melchor, M.T.; Montaño, J.G.; Díaz, F.J., \& Cervantes, F. (2013). Desarrollo y validación de una ecuación para estimar el consumo máximo de oxígeno en niños de Secundaria en una prueba de un kilómetro. Revista Española de Educación Física y Deportes, 401, 12-19.

Mills, K.L.; Lalonde, F.; Clasen, L.S.; Giedd, J.N., \& Blakemore, S.J. (2014) Developmental changes in the structure of the social brain in late childhood and adolescence. Social Cognitive and Affective Neuroscience, 9(1), 123-131. doi: 10.1093/scan/nss113

Molero, D.; Ortega, F.; Valiente, I., \& Zagalaz, M.L (2010). Estudio comparativo del autoconcepto físico en adolescentes en función del género y del nivel de actividad físico-deportiva. Retos. Nuevas tendencias en Educación Física, Deprote y Recreación, 17,38-41.

Mota, J.; Santos, R.M.; Silva, P.; Aires, L.; Martins, C., \& Vale, S. (2012). Associations between self-rated health with cardiorespiratory fitness and obesity status among adolescent girls. Journal of Physical Activity and Health, 9(3),
378-381.

Ortega, F.B.; Ruiz, J.R.; Castillo, M.J., \& Sjöström, M. (2007). Physical fitness in childhood and adolescence: a powerful marker of health. International Journal of Obesity, 32(1), 1-11. doi:10.1038/sj.ijo.0803774

Owen, N.; Salmon, J.; Koohsari, M.J.; Turrell, G., \& Giles-Corti, B. (2014). Sedentary behaviour and health: mapping environmental and social contexts to underpin chronic disease prevention. British Journal of Sports Medicine, 48(3), 174-177. doi:10.1136/bjsports-2013-093107

Parschau, L.; Fleig, L.; Koring, M.; Lange, D.; Knoll, N.; Schwarzer, R., \& Lippke, S. (2013). Positive experience, self-efficacy, and action control predict physical activity changes: A moderated mediation analysis. British Journal of Health Psychology, 18(2), 395-406. doi: 10.1111/j.2044-8287.2012.02099.x

Pastorelli, C.; Caprara, G.V.; Barbaranelli, C.; Rola, J.; Rozsa, S., \& Bandura, A. (2001). The structure of children's perceived self-efficacy: a cross-national study. European Journal of Psychological Assessment, 17(2), 87-97. doi: 10.1027// 1015-5759.17.2.87

Pesce, C.; Faigenbaum, A.; Crova, C.; Marchetti, R., \& Bellucci, M. (2013). Benefits of multi-sports physical education in the elementary school context. Health Education Journal, 72(3), 326-336. doi: 10.1177/0017896912444176

Pontifex, M.B.; Raine, L.B.; Johnson, C.R.; Chaddock, L.; Voss, M.W.; Cohen, N.J.; Kramer, A.F., \& Hillman, C.H. (2011). Cardiorespiratory fitness and the flexible modulation of cognitive control in preadolescent children. Journal of Cognitive Neuroscience, 23(6), 1332-1345. doi:10.1162/jocn.2010.21528

Ramírez-Lechuga, J.; Femia, P.; Sánchez-Muñoz, C., \& Zabala, M. (2011). La actividad física en adolescentes no muestra relación con el consumo máximo de oxígeno. Archivos de Medicina del Deporte, 28(142), 103-112.

Raudino, A.; Fergusson, D.M., \& Horwood, L.J. (2013). The quality of parent child relationships in adolescence is associated with poor adult psychosocial adjustment. Journal of Adolescence, 36(2), 331-340. doi: 10.1016/ j.adolescence.2012.12.002

Shavelson, R.J.; Hubner, J.J., \& Stanton, J.C. (1976). Self-concept: validation of construct interpretations. Review of Educational Research, 46(3), 407-441.

Slutzky, C.B., \& Simpkins, S.D. (2009). The link between children's sport participation and selfesteem: exploring the mediating role of sport self-concept. Psychology of Sport and Exercise, 10(3), 381-389. doi: 10.1016/ j.psychsport.2008.09.006

Spilt, J.L.; Lier, P.A.; Leflot, G; Onghena, P., \& Colpin, H. (2014). Children's Social Self-Concept and Internalizing Problems: The Influence of Peers and Teachers. Child Development, 85(3), 1248-1256. doi: 10.1111/cdev.12181

Tomporowski, P.; Davis, C.; Miller, P., \& Naglieri, J. (2008). Exercise and Children's Intelligence, Cognition, and Academic Achievement. Educational Psychology Review, 20(2), 111-131. doi: 10.1007/s10648-007-9057-0

Vedul-Kjelsäs, V.; Sigmundsson, H.; Stensdotter, A.K., \& Haga, M. (2011). The relationships between motor competence, physical fitness and self-perception in children. Child: Care, Health and Development, 38(3), 394-402. doi: 10.1111/ j.1365-2214.2011.01275.x

Velez,A.; Golem, D.L., \&Arent, S.M. (2010). The impact of a 12-week resistance training program on strength, body composition, and self-concept of Hispanic adolescents. Journal of Strength and Conditioning Research, 24(4), 10651073. doi: 10.1519/JSC.0b013e3181cc230a

Viholainen, H.; Aro, T.; Purtsi, J.; Tolvanen,A., \& Cantell, M. (2014). Adolescents' school-related self-concept mediates motor skills and psychosocial well-being. British Journal of Educational Psychology, 84(2), 268-280. doi: 10.1111/ bjep.12023

Weinberg, R.S., \& Stockham, J. (2000). The importance of analyzing positionspecific self-efficacy. Journal of Sport Behaviour, 23(1), 60-69.

Wilson, H.E.; Siegle, D.; McCoach, D.B.; Little, C.A., \& Reis, S.M. (2014). A Model of Academic Self-Concept Perceived Difficulty and Social Comparison Among Academically Accelerated Secondary School Students. Gifted Child Quarterly, 58(2), 111-126. doi: 10.1177/0016986214522858

Zimmerman, B.J.(2000). Self-efficacy: An essential motive to learn. Contemporary Educational Psychology, 25(1), 82-91. doi: 10.1006/ceps.1999.1016

Zschucke, E.; Gaudlitz, K., \& Ströhle,A. (2013). Exercise and physical activity in mental disorders: clinical and experimental evidence. Journal of Preventive Medicine and Public Health, 46(Suppl 1), S12-S21. doi: 10.3961/ jpmph.2013.46.S.S12

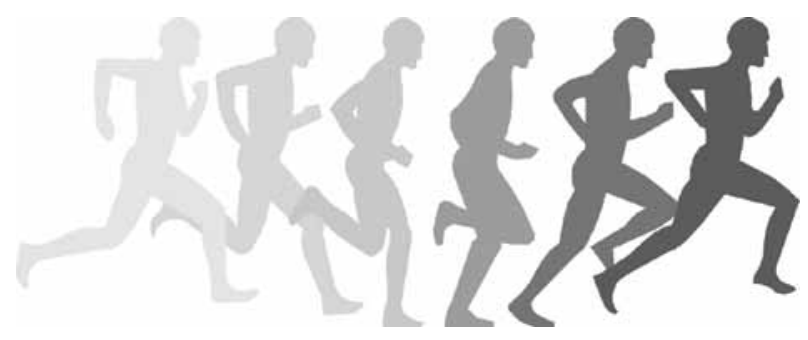

